\title{
Conference summary
}

\author{
Yasuo Fukui \\ Department of Physics and Astrophysics, Nagoya University, \\ Furo-cho, Chikusa-ku, Nagoya, Aichi 464-8602, Japan \\ email: fukui@a.phys.nagoya-u.ac.jp
}

\begin{abstract}
This is a summary of the highlights of the IAU Symposium 292, "Molecular Gas, Dust and Star Formation in Galaxies".
\end{abstract}

Keywords. galaxies: ISM — ISM: clouds — stars: formation

\section{Introduction}

The symposium covered topics related to gas physics and star formation in the Milky Way, nearby galaxies, and the distant universe. The motivation of the symposium was to bring together the experts on the subject both in the Galaxy and the external galaxies and to exchange ideas covering the low-redshift to high-redshift Universe. Molecules have been detected in various galaxies including spirals, ellipticals, and dwarfs, offering a powerful tool to diagnose the interstellar medium (e.g., talks by Wilson, Bureau, Wang). A number of excellent papers were presented in the symposium and it is impossible to cover all of them in this brief review. I will therefore focus on some of the selected results which attracted the reviewer's attention. The review given at the end of the symposium was a personal reflection on the papers presented.

The main issues addressed may be summarized as follows as given in the plenary invited review by Mac Low; these also give a balanced view of the main topics of the meeting:

- Star formation rate is low at high $z$. Is this due to feedback?

- Does turbulence inhibit star formation?

- $\mathrm{H}_{2}$ correlates with star formation but doesn't cause it.

- Dust shielding is needed for star formation.

- Gravitational instability drives star formation.

The following additional issues may also be included:

- What is the dark gas: molecular or atomic?

- What is the role of magnetic fields?

- Do very dense clumps, e.g., infrared dark clouds (IRDCs), really form stars?

- What is the role of triggering in star formation? For instance, are cloud-cloud collisions important in star formation?

\section{Giant molecular clouds}

Giant molecular clouds (GMCs) are the densest phase of the interstellar medium (ISM) and are therefore supposed to be the primary site of star formation. Only a small fraction of GMCs are, however, forming stars, and the star formation efficiency is very low 
in GMCs. The major issues on GMCs are as follows (e.g., talk by Tan):

- GMCs form stars.

- Star formation is inefficient in GMCs.

- If GMCs live longer than the free fall timescale, $t_{\mathrm{ff}}$, what maintains turbulence?

- Are GMCs transient and unbound?

- How are GMCs formed and destroyed?

The GMC mass range is $10^{4}-10^{7} M_{\odot}$ (Williams et al. 2000). Higher-mass GMCs of $10^{5}-10^{6} M_{\odot}$ number about 3000 in the Galaxy, while lower-mass GMCs of $10^{4}-10^{5} M_{\odot}$ are more numerous, $10^{5}$ clouds in the Galaxy. Perhaps both types of GMCs are important in forming stars, although high-mass GMCs have been more often the subject of past studies.

An overview of giant molecular clouds (GMCs) was given in the beginning of the symposium (talk by Tan). GMCs are perhaps close to the virial equilibrium and a small fraction of dense cores in GMCs form stars. GMCs have supersonic turbulence whose origin is not yet well understood. Feedback from stars is not so important in driving turbulence. Self-gravity may drive turbulence or the initial motion of lower-density converging flows may survive as the turbulent motions in GMCs. It is not necessarily proven that GMCs are gravitationally relaxed, and the filamentary shape of GMCs may suggest that the GMCs are not fully relaxed over a typical evolutionary timescale of $10 \mathrm{Myr}$ (Blitz et al. 2007; Fukui \& Kawamura 2010). The mass surface density may be a key factor in star formation. Infrared dark clouds (IRDCs) having mass density similar to GMCs are good candidates for high-mass star formation. The role of IRDCs in high-mass star formation is one of the key issues for the future.

Evolutionary pictures of GMCs were discussed in several talks. A model of GMC evolution in three steps from the quiescent phase of no $\mathrm{O}$ stars to the active phase of cluster/Hi region formation was discussed (talk by Goldbaum). In the LMC, Kawamura et al. (2009) classified GMCs detected in the NANTEN CO survey into three types according to the level of star formation activity [Type I, no O star formation; Type II, a few $\mathrm{O}$ stars formed; Type III, active formation of rich clusters and HiI regions]. More recently, a high resolution CO survey of M 33 with the IRAM 30 m telescope has covered the whole galactic disk and resolved 337 GMCs at 40 pc resolution, allowing one to make a comparison with young high-mass stars (talk by Braine). This study also finds that GMCs can be grouped into similar types as found in the LMC. A new CO survey of M 51 presented continuous CO spiral arms which are not yet resolved into individual GMCs in this ISM-rich spiral. GMCs are often embedded in HI gas. Formation of GMCs is likely taking place from Hi gas (Fukui et al. 2009). Accretion of Hi gas to form GMCs may drive turbulence and make the lifetime of GMCs longer than $t_{\mathrm{ff}}$. Three types of HI-to- $\mathrm{H}_{2}$ transition regions - the LMC, the Galaxy and M 51 - were discussed; in M 51, GMCs are tightly connected to $\mathrm{CO}$ with little $\mathrm{HI}$, but the conversion of $\mathrm{HI}$ into $\mathrm{H}_{2}$ seems important in the HI-rich LMC (talk by Elmegreen).

Dense clumps in GMCs are the sites of star formation. Sub-mm multi- $J$ transitions of $\mathrm{CO}$ are used to derive detailed physical parameters of such clumps with sub-mm telescopes (ASTE and NANTEN2) and showed that more evolved GMCs have denser and warmer clumps (talk by Kawamura). This suggests that a GMC and its internal clumps evolve toward a denser phase on a time scale of $10 \mathrm{Myr}$. These studies will be extended into higher resolution studies with ALMA and will provide an unprecedented detailed and unbiased view of GMC evolution in the nearest galaxies. 


\section{Filamentary distribution of the ISM}

Molecular clouds are not round. The Herschel satellite resolved Galactic infrared dust emission into numerous filaments and dense clumps and the dust emission corresponds to molecular clouds. The clumps have surface mass density of $1-10 \mathrm{~g} \mathrm{~cm}^{-2}$, which may be preferentially connected to the filaments, suggesting that filaments play a key role in forming dense cores (Molinari et al. 2010). ALMA Cycle 0 observations have shown that in one of the IRDCs, SDC 335, the mass infall rate onto the core is very high, $\sim 10^{-3} M_{\odot} / \mathrm{yr}$, along the filaments connected to the core (talk by Fuller). The filaments are very quiescent with $1 \mathrm{~km} \mathrm{~s}^{-1}$ velocity differences and no velocity gradient in each filament. It is of particular interest to resolve more IRDC cores to better establish the high mass accretion rate. In spite of such inflow signatures, it is surprising that many of the IRDCs show little sign of star formation. Another filamentary IRDC, G035.39-00.33, may consist of two filaments merging to form young stars (talk by Caselli).

\section{Star formation laws}

The Kennicutt-Schmidt law relates the gas surface density $\Sigma$ and the star formation rate (SFR) in GMCs. Significant details of the star formation laws including the starburst sequence and disk sequence are now available (e.g., Daddi et al. 2010; Bigiel et al. 2008). The basic trend is defined by the disk sequence, while the starburst galaxies show an order of magnitude higher SFR. The $\Sigma-M$ diagram may offer another useful tool to diagnose star formation properties in various clouds (talk by Tan). It was also emphasized that extinction is the key factor controlling star formation and that molecules are not playing that role (talks by Mac Low and Krumholz).

The importance of triggered star formation was not much discussed in the symposium. It was noted that triggering may not be a major mechanism of star formation (review by Mac Low), while triggering signatures including the Spitzer bubbles nonetheless suggest a role for triggering (poster by the Purple Mountain Observatory group) and collisions between clouds are discussed as a promising major mode of star formation (talk by Tan). Recent findings of collision induced star formation in Westerlund 2 and M20 lend support for the latter view (e.g., Furukawa et al. 2009; Torii et al. 2011). The net effects of triggering remain as a major challenge in better understanding star formation, particularly at the high-mass end.

\section{High- $J$ CO transitions: ISM diagnostics of GMC clumps}

Studies of individual GMCs offer insight into more details of star formation. It was impressive that new instruments have broadened spectroscopy and photometry over a large wavelength coverage (Herschel, SOFIA, AKARI, and ALMA). Thanks to the widened wavelength coverage and higher spectral resolution we are able to observe multi- $J$ transitions of $\mathrm{CO}$ and other molecules, a key to model physical and chemical conditions of the ISM. In Mrk 231, many high- $J$ transitions of CO in the far infrared have been detected (talk by Papadopoulos). Vibrationally excited transitions of HCN have been detected in the active galactic nucleus of 3C273 (talk by Sakamoto). These new tools have started to provide a reliable determination of density and temperature, allowing us to refine our understanding of heating and cooling as well as ISM chemistry. 


\section{Dark gas}

It is important to measure the ISM mass in estimating star formation efficiency. The high sensitivity of ALMA has been used to accurately measure the dust component of the ISM mass in starburst galaxies (talk by Scoville).

Dark gas is the ISM which cannot be traced either by the $\mathrm{CO}$ or $\mathrm{HI} \mathrm{mm} / \mathrm{cm}$ transitions and perhaps corresponds to an intermediate density range from 100 to $10^{3} \mathrm{~cm}^{-3}$. The prevalence of dark gas was shown in the local ISM through a comparison between radiodetected ISM and the whole sky $\gamma$-ray distribution (Grenier et al. 2005), after some observational and theoretical works had suggested or predicted such a component (e.g., Madden et al. 1997). The recent widened wavelength coverage makes it possible to explore further the nature of the dark gas. Dust continuum by Planck measured the dark gas in the same local ISM (Planck Collaboration 2011). Dust in the LMC, the SMC, and the Local Group galaxies also suggest that the dark gas is common in these galaxies (Bot et al. 2010; Roman-Duval et al. 2010; Galliano et al. 2011; Leroy et al. 2011; Bolatto et al. 2011).

[CII] is another possible indicator of the dark gas (talk by Velusamy). High Cir/CO ratios in dwarf galaxies like IC 10 indicate that low metallicity CO-dark $\mathrm{H}_{2}$ gas may be important (talk by Madden). Also the SMC shows a sub-mm excess which is not explicable by the current knowledge of dust properties (talk by Rubio).

Another possibility raised recently is the importance of opaque HI gas on the galactic scale in M33 and the LMC (talk by Braun). For the Hi column density range around $10^{21}$ $\mathrm{cm}^{-2}$, the optical depth of the $21 \mathrm{~cm} \mathrm{Hi} \mathrm{transition} \mathrm{may} \mathrm{become} \mathrm{greater} \mathrm{than} \mathrm{unity} \mathrm{and} \mathrm{is}$ not accurately estimated by the usual optically thin approximation (Fukui 2013). Such dense and cool Hi gas is also detected in the $\gamma$-rays in a SNR and the Galactic Center and may be a good candidate for the "atomic" dark gas, an alternative to the molecular dark gas (Fukui et al. 2012; Hayakawa et al. 2012).

\section{Dust}

The AKARI satellite made an infrared survey of the whole sky at infrared wavelengths, which is complementary to the Herschel observations (talk by Onaka). The AKARI survey is useful in assessing the SED of various galaxies, and spatially resolved infrared SEDs probe the size distribution of dust and the formation sites of each component including PAH, VSG, and BG. Diagnoses of the ISM by the relative strengths of unidentified infrared (UIR) features are in progress (e.g., Mori et al. 2013). The 3.3 micron band gives critical information on the dust size distribution and relative abundance, key knowledge on the formation and destruction process of dust in shocked regions (Cyg loop: Sankrit et al. 2010; Pup A: Arendt et al. 2010; Andersen et al. 2011). UIR band carriers in BCDs are suggested to be large, with small carriers having been destroyed. The 24 micron AKARI survey observed mass loss from carbon stars. The ejected carbon may become graphite or PAH which might lead to formation of pre-biotic material (talk by Kaneda). The origin of dust may be explained by AGN and SN, whereas dust grown in the ISM must be dominant to fit the total ISM dust emission (talk by Gomez).

\section{Galactic centers and nuclear outflows}

The central regions of galaxies show cloud properties significantly different from in the disk (e.g., M64; talk by Blitz). An extensive molecular line survey of the Central Molecular Zone (CMZ) has been completed with the Mopra $22 \mathrm{~m}$ telescope and the 
violent motion and complicated rich molecular chemistry in the CMZ are now emerging (talk by Burton). Herschel observed a dust ring of 100 pc radius associated with the CMZ, which was modeled by elliptical orbits surrounding the central stellar bar (talk by Molinari). The violent motion is not only toward the CMZ but also at a kpc scale from the center. The foot points of magnetic flotation loops (loops 1 and 2, Fukui et al. 2006) also show shocked gas properties as indicated by the decrease of PAH features through AKARI observations (talk by Kaneda).

In the centers of external galaxies, gas motion appears related with active star formation. The central region of the molecular disk in NGC 253 shows bubble-like features possibly driven by young star clusters (talk by Matsushita). A gigantic CO web extending as far as $50 \mathrm{kpc}$ from the active nucleus is traced by CO in NGC 1275 and evidence for a high velocity outflow in the center of Mrk 231 has been revealed (talk by Salome). Although the origin of such structures is not yet fully understood, they are perhaps related to the active starbursts in the centers of the galaxies (Feruglio et al. 2010).

\section{In the end}

Many new and exciting results covering a wide range of topics on star formation in galaxies were presented in this symposium. I thank all the participants for their invaluable contributions and do hope that the symposium becomes a valuable step forward to enhance exchange and to promote future research in the field.

\section{References}

Andersen, M., Rho, J., Reach, W. T., Hewitt, J. W., \& Bernard, J. P. 2011, ApJ 742, 7

Arendt, R. G., Dwek, E., Blair, W. P., et al. 2010, ApJ 725, 585

Bigiel, F., Leroy, A., Walter, F., et al. 2008, AJ 136, 2846

Blitz, L., Fukui, Y., Kawamura, A., et al. 2007, Protostars and Planets V 81

Bolatto, A. D., Leroy, A. K., Jameson, K., et al. 2011, ApJ 741, 12

Bot, C., Ysard, N., Paradis, D., et al. 2010, A\&A 523, A20

Daddi, E., Elbaz, D., Walter, F., et al. 2010, ApJ (Letters) 714, L118

Feruglio, C., Maiolino, R., Piconcelli, E., et al. 2010, A\&A (Letters) 518, L155

Fukui, Y., Yamamoto, H., Fujishita, M., et al. 2006, Science 314, 106

Fukui, Y., Kawamura, A., Wong, T., et al. 2009, ApJ 705, 144

Fukui, Y., Sano, H., Sato, J., et al. 2012, ApJ 746, 82

Fukui 2013, in preparation

Fukui, Y. \& Kawamura, A. 2010, ARAA 48, 547

Furukawa, N., Dawson, J. R., Ohama, A., et al. 2009, ApJ (Letters) 696, L115

Galliano, F., Hony, S., Bernard, J.-P., et al. 2011, A\& $A$ 536, A88

Grenier, I. A., Casandjian, J.-M., \& Terrier, R. 2005, Science 307, 1292

Hayakawa, T., Torii, K., Enokiya, R., Amano, T., \& Fukui, Y. 2012, PASJ 64, 8

Kawamura, A., Mizuno, Y., Minamidani, T., et al. 2009, ApJS 184, 1

Langer, W. D., Velusamy, T., Pineda, J. L., et al. 2010, A\&广A (Letters) 521, L17

Leroy, A. K., Bolatto, A., Gordon, K., et al. 2011, ApJ 737, 12

Madden, S. C., Poglitsch, A., Geis, N., Stacey, G. J., \& Townes, C. H. 1997, ApJ 483, 200

Molinari, S., Swinyard, B., Bally, J., et al. 2010, A\&SA (Letters) 518, L100

Mori et al. 2013, in preparation

Planck Collaboration, Ade, P. A. R., Aghanim, N., et al. 2011, A $\& A$ 536, A19

Roman-Duval, J., Israel, F. P., Bolatto, A., et al. 2010, A $₫ A$ (Letters) 518, L74

Sankrit, R., Williams, B. J., Borkowski, K. J., et al. 2010, ApJ 1092

Torii, K., Enokiya, R., Sano, H., et al. 2011, ApJ 738, 46

Williams, J. P., Blitz, L., \& McKee, C. F. 2000, Protostars and Planets IV 97 